\title{
Research on Noise Control of Product Symbol Communication Channel-Take a Small Rice Mill for Example
}

\author{
Zaiyao SHEN, Rui WU, Lu CHEN, and Qiong WU \\ College of Art and Design, Nanjing Tech University
}

\begin{abstract}
With the in-depth application of semiotics in product design, it has brought great changes to modern design process. Starting from the noise nodes generated in the product design process, taking the actual case of a small rice mill as an example, starting from the aspects of structure, semantics and pragmatics, through the combination of theory and practice. Finally, the feasibility and practical value of noise control in symbol transmission channel are verified.
\end{abstract}

Keywords. Semiotics, A channel, Noise control

\section{Overview of product symbol communication research}

\subsection{Symbol transmission mode}

Symbol is the carrier of expressing information, and symbol design of products is a systematic way for designers to convey their own design ideas to consumers. However, with the development of electronization and integration, the mechanical constraints on the form of products are gradually reduced, which blurs the boundaries of products to a certain extent. To some extent, the traditional "form following function" is no longer unique.

The application of semiotics has greatly changed product design. With the application of semiotics, design activities are no longer just a personal show of designers' abilities, and product design has gradually become systematic and standardized. Designers have their own "password book", and communicate their ideas contrast this "password book", which is widely recognized by consumers. Combined with the product design process, Figure 1 is the process of product symbolcommunication.

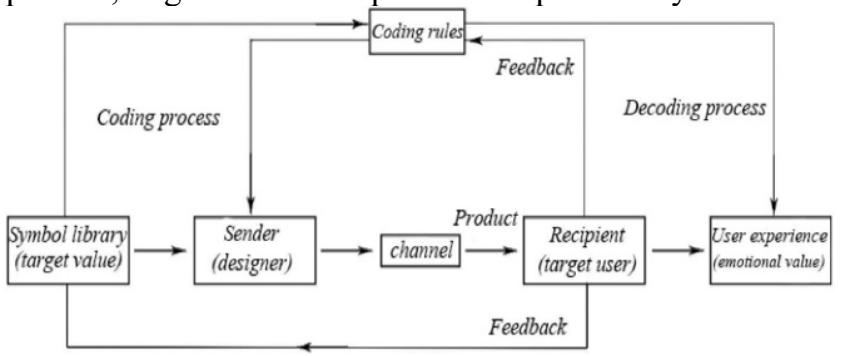

Figure 1. Symbol communication flow 


\subsection{Channel noise analysis}

Physiologically, noise refers to all sounds that cause discomfort or endanger people's physical and mental health physically, noise refers to all irregular signals (not necessarily sounds). In the field of semiotics, noise can be roughly divided into two types, namely mechanical noise and ambiguous noise.

Traditional mechanical noise is mainly concentrated in the landing process of products. After the design scheme is determined, the final finished product may be quite different from the designer's original scheme due to the unstable factors in various processing links such as process. Therefore, a complete product development team should be equipped with modeling designers, structural engineers, process engineers, UI designers, etc., and production supporting engineers are indispensable. At the same time, the project team should participate in the front-line production when conditions permit, so as to reduce some useless ideas that are out of the practical sense.

Different from mechanical noise, ambiguous noise appears in the whole channel process. In the symbol transmission mode, the sender is usually a designer. In the scheme design stage, because each designer has different professional abilities and cognitive levels, if the team lacks good cooperation and communication, there will be differences within the team, and the channel will generate noise from the initial stage.In the whole process of communication, the noise will become louder and louder, which blurs the information that the sender wants to transmit to the recipient.

With the advent of the era of big data, network communication has become the most common channel. At the same time, big data also makes human information storage four times faster than the growth rate of the world economy, and now the data can be doubled every three years. At that same time, however, a large amount of data is naturally accompanied by a large amount of junk data, Misunderstanding will inevitably occur in the process of receiving fragmented data, so the ability to get the information you need from it and understand it smoothly will become more and more important.

The addressees in symbol transmission mode are generally product users. However, users of products may come from different regions and countries, they have different educational levels and cognitive abilities, and their cultures are mostly different. Therefore, the sender needs to know the culture of the target user before sending the information, Avoid the target user's aversion due to cognitive errors.

\section{Noise control method of product symbol transmission}

System completion can effectively reduce the mechanical and ambiguous noises of symbols in the process of channel transmission. Therefore, designers should base themselves on the whole product system engineering and pay attention to the coding specifications of symbols in different design stages.

\subsection{Linguistic noise control methods}

In order to effectively realize the coordination among man, machine and environment, prevent noise interference, and ensure the accuracy of later modeling design and semantic transmission. In the stage of product conceptual design, we should focus on inproduct system engineering, and use the principles of linguistics to realize the organic communication between each subsystem and the outside world. On the practical level, 
after accepting the design task. Design research must include market research, user demand research, technical research, modeling rules and other links. The research link must adhere to the principles of scientific, systematic and objective timeliness. Finally, according to the research product symbol information, the relevant information should be carefully screened and screened, and the corresponding coding rules should be designed. Finally, the content conveyed by the product symbol should be clarified. That is, determine the product positioning; Realize the semantics of products accurately through the design of product modeling. Taking a small household rice mill as an example, the selection of its target consumer group requires designers to substitute the user's perspective and design products that meet the user's needs from the user's perspective. The user group in this example is clear. That is to say, individuals and families who pursue the way of health care, as well as restaurants that play the cards of healthy eating, etc.

The core technologies of household rice milling machine are intelligent precision control technology and sheet metal forming technology. The former is the technology for realizing its core functions, while the latter is an important technology for its processing and manufacturing. The materials and processing technologies of its different subsystems are shown in Table 1.

Table 1. Processing processes corresponding to different components of each system

\begin{tabular}{|c|c|c|c|}
\hline Name & Material & Affiliated Module & Processing Technology \\
\hline Die casting & Cast iron & Mechanical system & $\begin{array}{l}\text { Pressure casting, numerical } \\
\text { control }\end{array}$ \\
\hline Machine body shell & $\begin{array}{l}\text { Cold-roll steel } \\
\text { sheets }\end{array}$ & Mechanical system & $\begin{array}{c}\text { Cutting, blanking, bending and } \\
\text { pressing }\end{array}$ \\
\hline pulley & Rubber, PP plastic & Mobile system & $\begin{array}{l}\text { Michelin C3M technology, } \\
\text { injection molding }\end{array}$ \\
\hline Wheel hub & Aluminum alloy & Mechanical structure & Stamping and plastic forming \\
\hline Separator & Carbon steel & Tubing & Stamping \\
\hline
\end{tabular}

In order to meet the market demand, the main functions of domestic small rice mills need to meet the following requirements:

- Moveable;

- Miniaturization and lightweight;

- High precision machining;

- Visualization operation;

- One-key fool operation.

With the progress of society, the complicated design style has been abandoned by the times, and the mainstream style in the world is the simplification of operation, the simplification of modeling, the miniaturization of size and the integration of functions.

Product design is inseparable from modeling design. In order to reduce the noise in the process of symbol information transmission, it is necessary to coordinate the relationship between the two in design. The modeling orientation of the rice mill in this example is as follows:

- The sense of science and technology reflects the breath of life;

- There are no unnecessary decorations and curved surfaces, and the functional operation is simple and straightforward;

- Color is combined with different decoration styles, with demand as the standard, and there is no clear standard.

After completing the product-related investigation, the design entered the stage of modeling design. In this stage, the designer should reduce the noise in the channel process by refining symbols from the consumer's point of view. At this stage, designers 
need to master channel technology, that is, to understand the chemical and physical properties of related materials and the processing technology and structural requirements of related products. It is impossible to completely remove noise, but designers can try their best to reduce noise interference.

\subsection{Semantic noise control methods}

Design team members come from all over the world, and each person has different specialties and cognitive abilities. Therefore, a team needs a product manager to organize these people together, so as to promote each team member to play their role actively and make the whole project go on in an orderly way.

In the preparation stage of the project, designers need to know the relevant product information. Nowadays, although there is many online information, it is disorganized and mostly lacks basis and timeliness. In order to obtain accurate information, apart from online information collection and processing, on-the-spot investigation, on-site visit and questionnaire interview are still indispensable for designers. Through careful preparation, investigation and information screening, the product positioning can be established effectively and accurately.

In the project design stage, the 'Three-Three-Three' conception advancing method is adopted, that is, three days to determine the direction, three days to sketch the conception and three days to determine the final plan. Taking the rice mill as an example, Figure 2 is the sketch conception at this stage.

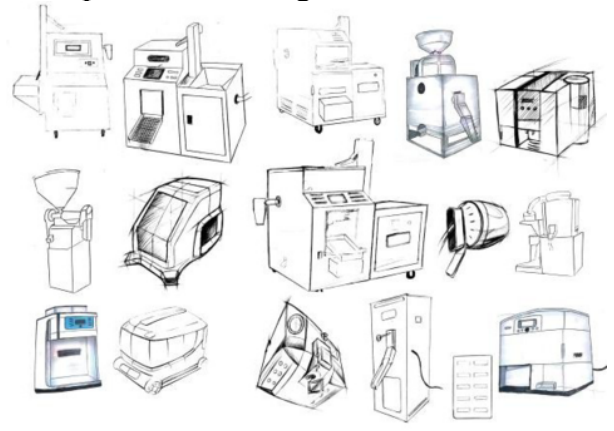

Figure 2. Sketch summary

At this stage, a large number of schemes will be eliminated. At this time, a reasonable and effective evaluation mechanism will play a huge role, which can effectively control the symbolic information and steadily advance the process of schemes. At this stage, designers need to keep more communication and brainstorm to make up for the blind spots in thinking. And finally determine the final plan (as shown in Figure 3)

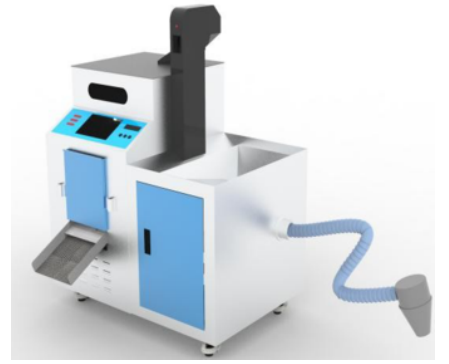

Figure 3. Final plan 


\subsection{Pragmatics noise control methods}

The research object of design pragmatics is the meaning expressed by design symbols in different contexts [1-3]. However, the composition of product symbols is not static, and the measurement standards of different products are different. It is difficult for designers to accurately convey the "artistic conception" expressed by design symbols to the audience, which is influenced by the communication context besides the deviation of design and processing. With the advent of the information age and the rapid expansion of the media industry, the dissemination of symbolic information is no longer directly conveyed by designers to consumers. Due to the division of labor between designers and marketing communicators, especially the existence of some unscrupulous public relations with false propaganda, the noise in the process of information dissemination tends to expand.

\subsubsection{Application of man-machine in design}

With the popularity of "people-oriented" design concept and the in-depth study of consumer psychology, in order to be recognized by the wider consumer groups, people find that only integrating people, machines and environment as a whole, implementing humanized design concept and establishing harmonious relationship between man and machine are the development direction [4-6]. Combined with the design environment, the man-machine orientation of this example is as follows:

- The height of the operation interface should be convenient for most adults in China to stand and operate;

- Because there is no limit to the degree of using group knowledge, its operation interface needs to be simple and easy to operate;

- The height of rice dumping area should be slightly higher than the height of adult crotch, which is convenient for grabbing and dumping rice.

\subsubsection{Symbol and code co-construction feedback system}

We can try to solve this problem through the co-construction of symbols, that is, coding the rules through the direct value of products, the inherent meaning of culture and the emotional experience value, and creating a common system through context, so as to maintain symbol interaction, so that consumers can also communicate in reverse and maintain effective two-way communication. So as to fundamentally reduce various noises in the transmission process.

\subsubsection{QFD application of QFD method in design evaluation}

In view of this kind of phenomenon, we can try to introduce six principles of aesthetics, and comprehensively cover the content of design evaluation from multiple dimensions. Considering the needs of users, the characteristics of products and the previous research on symbol positioning and coding, Finally, it is determined that the main factors affecting the communication effect of rice mill design are "convenient operation", "durable", "small and light weight", "position movable", "beautiful appearance", "moderate price" and "harmonious man-machine environment", which are taken as evaluation indexes. Here, QFD method (house of quality) is used for relevant evaluation. Figure 4 is the selected three competing products in the market. 


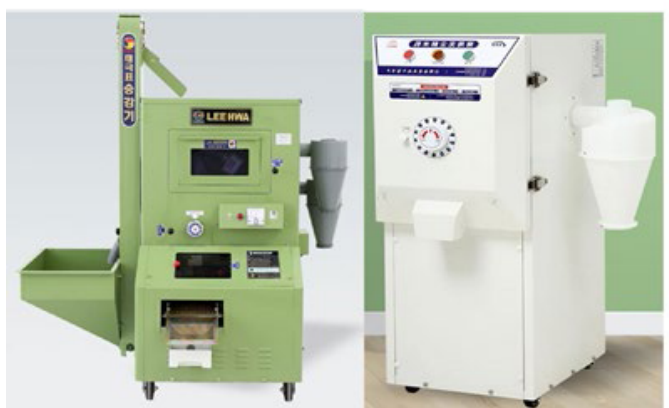

Competitive product 1 Competitive product 2

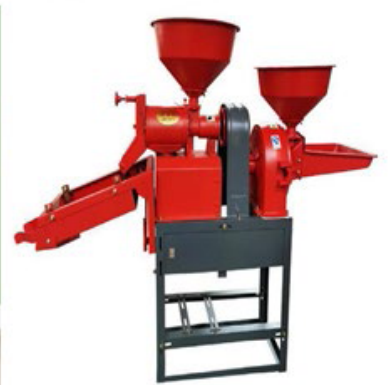

Competitive product 3

Figure 4. Comparison of competing products

Among them, the importance is scored quantitatively by the tested user, with a value of 1-5 points. The higher the score, the greater the importance, and the correlation is expressed by $\bigcirc$, in which has the highest correlation and $\bigcirc$ has the lowest correlation, and the weights are located at 1.5,1.2 and 1 respectively. The final house of quality is shown in figure 5.

\begin{tabular}{|c|c|c|c|c|c|c|c|c|c|c|}
\hline Consuser deand & $\begin{array}{c}\text { Importance } \\
\text { B }\end{array}$ & $\begin{array}{c}\text { Shell } \\
\text { material }\end{array}$ & Noise index & $\begin{array}{c}\text { Color } \\
\text { selection }\end{array}$ & $\begin{array}{l}\text { Operation } \\
\text { sode }\end{array}$ & $\mid \begin{array}{c}\text { Operation } \\
\text { panel layout }\end{array}$ & $\begin{array}{l}\text { Design } \\
\text { scheree }\end{array}$ & $\begin{array}{c}\text { Competitive } \\
\text { products } 1\end{array}$ & $\begin{array}{l}\text { Competitive } \\
\text { products } 2\end{array}$ & $\begin{array}{l}\text { Competitive } \\
\text { products } 3\end{array}$ \\
\hline $\begin{array}{l}\text { Convenient } \\
\text { operation }\end{array}$ & 5 & & & & $\bullet$ & 0 & 3.8 & 3.5 & 3.2 & 4 \\
\hline Durable & 4 & $\bullet$ & & & 0 & & 3.1 & 4 & 3.2 & 3.8 \\
\hline Seall and lightreight & 2 & 0 & $\circ$ & & 0 & & 2.6 & 2.5 & 3 & 4 \\
\hline Position novable & 4 & & ० & & 0 & & 4.4 & 4. 2 & 4 & 3.6 \\
\hline Beautiful appearance & 4 & & & $\bullet$ & & & 4.1 & 3.7 & 3.9 & 2.5 \\
\hline Xoderate price & 5 & 0 & & & & & 3.6 & 3.7 & 3 & 4. 2 \\
\hline $\begin{array}{l}\text { Harnonious man- } \\
\text { machine environment }\end{array}$ & 3 & & $\bullet$ & 0 & & 0 & 3.0 & 3.1 & 3.6 & 2.2 \\
\hline
\end{tabular}

Figure 5. Evaluation of Quality House competitiveness

Market competitiveness index $=\mathrm{A} 1 \mathrm{~B} 1+\ldots+\mathrm{AnBn} /(\mathrm{B} 1+\mathrm{B} 2+\mathrm{B} 3+\ldots+\mathrm{Bn})$. Generation $\mathrm{A}$ refers to the score of user demand items, and generation $B$ refers to the importance of user demand items. The final finishing results are shown in Table 2.

Table 2. Product competitiveness comparison

\begin{tabular}{rrrrrr}
\hline Contrastive item & Design scheme & $\begin{array}{c}\text { Competing goods } \\
\mathbf{1}\end{array}$ & $\begin{array}{c}\text { Competinggoods } \\
\mathbf{2}\end{array}$ & Competinggoods 3 \\
\hline Market & 2.19 & 2.18 & 2.14 & 2.15 \\
Competitiveness & & & & \\
Index & 1 & 2 & 4 & 3 \\
Market & & & & \\
$\begin{array}{r}\text { Competitiveness } \\
\text { Ranking }\end{array}$ & & & & \\
\hline
\end{tabular}

Through calculation and comparison, although the market competitiveness of the design scheme ranks first, it is only slightly higher than the other three competitive products. It can only be said that it barely meets the requirements of users, but it is still far from solving the actual pain points of users. At the same time, it can be seen that the homogenization of related products is more serious at present. 


\section{Summary}

In the process of transmitting the symbolic information to the receiver, there is a deviation due to the existence of various noises in each link of the channel transmission, which seriously affects the communication of the designer's idea. In view of this kind of phenomenon, this paper starts from the aspects of structure, semantics and pragmatics, covering the whole process of channel transmission from prevention, positive solution to feedback, and combining with the example of domestic small rice mill, Different methods of channel noise reduction are summarized. In-depth analysis of the important role of designers in the process of product design, through the refinement and application of culture and customs to improve product design efficiency, while fully expressing design concepts. In addition, through the omni-directional study of the channel, from the source to the construction of the later feedback mechanism, Reducing the irregular noise in industrial production in an all-round way will eventually make modern industrial products better serve people.

\section{References}

[1] Wu Qiong, Jun Peng, Yu Zhang, Research on the paradigm of design pragmatics, Packaging Engineering, 37 (2016), 14:77-80.

[2] Qin Zhiguang, Liu Jiao, Liu Yao, Zhong Ting, Big Data Analysis Technology in Smart Cities. People's Post and Telecommunications Press: Academic Monograph on Information and Communication Innovation, 2015.

[3] Wu Qiong. Product System Design. Beijing: Chemical Industry Press, 2019

[4] Chen Xu, Semiotics Exploration in the Process of Industrial Design. Arts and Science, 32 (2019, 9:191192.

[5] Wu Qiong. Industrial design skills and taboos, Beijing: Mechanical Industry Press, 2009.8:46

[6] Deng Chaoming and Huang Yingxin. On the strategic transformation of local advertising companies under the background of integrated marketing communication-taking interchange International Communication Group as an example. Journal of China University of Geosciences (Social Science Edition), 14 (2014), 3:122-128. 